\title{
Editorial for the Special Issue on Financial Econometrics
}

\author{
Yiu-Kuen Tse \\ School of Economics, Singapore Management University, Singapore 178903, Singapore; yktse@smu.edu.sg
}

Received: 16 September 2019; Accepted: 17 September 2019; Published: 19 September 2019

Financial econometrics has developed into a very fruitful and vibrant research area in the last two decades. The availability of good data promotes research in this area, specially aided by online data and high-frequency data. These two characteristics of financial data also create challenges for researchers that are different from classical macro-econometric and micro-econometric problems.

This special issue is dedicated to research topics that are relevant for analyzing financial data. We have gathered six articles under this theme. The paper by Eriksson et al. (2019) considers a method to forecast realized volatility using a classical autoregressive model. Two modifications are adopted to make this model suitable for nonnegative valued variables like volatility. First, they apply Tukey's power transformation to their data. Second, they allow the error distribution to be unspecified, resulting in a semiparametric approach. While their model has forecasting volatility as the primary motivation, it can be used for many nonnegative valued variables, thus extending the applicability of their approach.

The empirical study of Eriksson et al. (2019) shows that their method compares very well against some of the most commonly used forecasting models for volatility in terms of post-sample prediction. As mentioned in their concluding remarks, it will be interesting to see how their approach works for intra-day data and multivariate models.

Ahmed and Satchell (2019) consider a threshold autoregressive model with Markovian states. These states may incorporate both explosive and stationary regimes. They investigate the characteristic function of this process and derive analytic formula for their moments. Their approach can be applied to processes for which the moment generating function does not exist. Thus, certain asset pricing models with non-normal errors can be analyzed.

Xiao and Sun (2019) investigate the estimation of the tuning parameter for model selection and averaging. Incorporating the shrinkage averaging estimator method and Mallow's model averaging method, they propose the shrinkage model averaging method, which can be used for averaging high-dimensional sparse models. The method is applicable to a wide range of econometric models, and extends beyond the financial econometrics arena. Their Monte Carlo study shows that their new method performs well against other methods in averaging high-dimensional sparse models.

Men et al. (2019) propose a threshold stochastic conditional duration model that can be used to analyze transaction financial data. They assume a latent AR(1) model, which may switch between two regimes. The regimes are self-excited and are based on the observed duration. The model can be estimated efficiently using a Markov-Chain Monte Carlo approach. Their empirical examples support the desirable performance of their new model in forecasting transaction duration.

Hevia and Sola (2018) examine the effect of imposing over-identifying restrictions on affine term structure models. In particular, they investigate the effects of inappropriate restrictions on some risk measures. They argue that in certain cases, such restrictions may have a significant impact on the estimated risk premium, and it is difficult to ascertain a priori the likely outcome. Due to this uncertainty, they recommend using just-identified models when the purpose is to apply the affine models to compute the risk premium. 
Grynkiv and Stentoft (2018) study the multivariate generalization of the threshold autoregressive model. They assume the latent regime driver to follow a dichotomous structure, and one of the regimes may be explosive. They derive conditions under which the overall distribution is stationary. They also derive the unconditional distribution of the process for a special case of the threshold model and show that it follows an infinite mixture-of-normal distribution.

Funding: This research received no external funding.

Conflicts of Interest: The author declares no conflict of interest.

\section{References}

Ahmed, Muhammad F., and Stephen Satchell. 2019. Some Dynamic and Steady-State Properties of Threshold Auto-Regressions with Applications to Stationarity and Local Explosivity. Journal of Financial Risk Management 12: 123. [CrossRef]

Eriksson, Anders, Daniel P. A. Preve, and Jun Yu. 2019. Forecasting Realized Volatility Using a Nonnegative Semiparametric Model. Journal of Financial Risk Management 12: 139. [CrossRef]

Grynkiv, Galyna, and Lars Stentoft. 2018. Stationary Threshold Vector Autoregressive Models. Journal of Financial Risk Management 11: 45. [CrossRef]

Hevia, Constantino, and Martin Sola. 2018. Bond Risk Premia and Restrictions on Risk Prices. Journal of Financial Risk Management 11: 60. [CrossRef]

Men, Zhongxian, Adam W. Kolkiewicz, and Tony S. Wirjanto. 2019. Threshold Stochastic Conditional Duration Model for Financial Transaction Data. Journal of Financial Risk Management 12: 88. [CrossRef]

Xiao, Hui, and Yiguo Sun. 2019. On Tuning Parameter Selection in Model Selection and Model Averaging: A Monte Carlo Study. Journal of Financial Risk Management 12: 109. [CrossRef]

(C) 2019 by the author. Licensee MDPI, Basel, Switzerland. This article is an open access article distributed under the terms and conditions of the Creative Commons Attribution (CC BY) license (http://creativecommons.org/licenses/by/4.0/). 